\title{
Benefícios socioeconômicos da adoção de novas tecnologias no cultivo do açaí no Estado do Pará1
}

\author{
Ana Karlla Magalhães Nogueira ${ }^{2 *}$, Antônio Cordeiro de Santana ${ }^{3}$ \\ 10.1590/0034-737X201663010001
}

\section{RESUMO}

O objetivo deste trabalho foi calcular os benefícios socioeconômicos gerados pela adoção das novas tecnologias, no cultivo racional do açaí, no Estado do Pará, no período de 2001 a 2009. Utilizou-se o resultado das equações de demanda e oferta do açaí, estimadas por Nogueira (2011), com a finalidade de se obterem as elasticidades-preço da demanda e da oferta, para, assim, se aferirem os retornos sociais e econômicos da adoção de novas tecnologias sobre a economia do mercado de açaí no Estado. Os resultados mostraram que, a partir de 2001, com a adoção de tecnologias inovadoras no cultivo racional do fruto, houve substancial aumento do benefício socioeconômico para a população paraense. Esse benefício atingiu o patamar de R \$ 25.763,79 mil, o que representou um incremento de 48,78\% em relação ao benefício obtido antes da adoção das novas tecnologias ( $\mathrm{R} \$ 13.195,50$ mil). Com relação à distribuição dos benefícios, os consumidores foram os principais beneficiários, com 71,86\% dos benefícios totais.

Palavras-chave: inovação tecnológica, açaí, sistema de produção, benefícios socioeconômicos.

\section{ABSTRACT}

\section{Socioeconomic benefits from the new technologies in the açaí cultivation in Pará State}

The objective of this study was to calculate the socio-economic benefits generated by the adoption of new technologies in the rational cultivation of açaí in the state of Pará, in the period 2001-2009. We used the results of açaí supply and demand equations estimated by Nogueira (2011) in order to get the price elasticities of supply and demand so as to assess the social and economic returns of adoption of new technologies on the açaí market economy in the state. Results showed that, from 2001 through the adoption of innovative technologies in the rational cultivation of fruit there was a substantial increase in the socioeconomic benefit to Pará population. That benefit reaching a level of $\mathrm{R} \$ 25.763,79$ mil, which represented an increase of $48.78 \%$ compared to the benefit obtained before the adoption of the technology, R $\$ 13.195,50$ mil. Regarding the distribution of benefits, consumers have been the main beneficiaries with $71.86 \%$ of the total benefits.

Key words: technological innovation, açaí, production system, socio-economic benefits.

'Esse trabalho é parte da dissertação de mestrado da primeira autora.

${ }^{2}$ Universidade Federal Rural da Amazônia, Instituto Socioambiental e dos Recursos Hídricos, Belém, Pará, Brasil. karlla_ufra@hotmail.com

${ }_{3}^{3}$ Universidade Federal Rural da Amazônia, Instituto Socioambiental e dos Recursos Hídricos, Belém, Pará, Brasil. antonio.acsantana@yahoo.com.br

*Autora para correspondência: karlla_ufra@hotmail.com
} 


\section{INTRODUÇÃO}

O Estado do Pará, com uma área de $1.253 .164,5$ km², aproximadamente $15 \%$ do território brasileiro, destaca-se no cenário nacional como maior produtor de açaí (Euterpe oleracea Mart.). Em 2013, a participação desse estado na produção brasileira de açaí fruto foi de $85,6 \%$, seguida pela do Maranhão, com 8,8\%, sendo os únicos estados a registrarem aumentos da produção desta fruta no país (IBGE, 2013). Os sistemas de produção extrativos tradicionais e extrativo manejado representam 79,28\% e os cultivos em terra firme, sem e com irrigação e, ou, em sistemas agroflorestais participam com 20,72\% (IBGE, 2006).

Até meados dos anos de 1990, o açaí era destinado, basicamente, ao consumo do mercado local, em especial pelos povos ribeirinhos e pelas camadas populacionais de baixa renda, tanto do meio rural quanto do urbano (Santana \& Costa, 2008).

Contudo, com a divulgação de suas qualidades energéticas e nutritivas,o açaí, rapidamente, passou a ser comercializado em supermercados, casas de suco, restaurantes, lanchonetes de academias e praias, ganhando a preferência dos adeptos da alimentação saudável em todo Brasil e no mercado internacional (Bobbio et al., 2000; Santana \& Gomes, 2005; Silva et al., 2006; Santana \& Costa, 2008; Teixeira et al., 2008).

A nova dinâmica do mercado do açaí, tanto local quanto nacional e internacional, tem-se caracterizado por uma demanda crescente, que contribui para o aumento dos preços, favorecendo a exportação, pois somente entre 2002 e 2009, o valor aumentou de US\$ 1,04 milhão para US\$ 24,0 milhões, respectivamente, em polpa congelada e pasteurizada, para EUA, Europa e Japão, reduzindo a oferta para o mercado nacional (Santana, 2007; Homma \& Santana, 2009; Nogueira, 2011).

Esse novo cenário, portanto, gerou uma mudança de atitude por parte dos extrativistas que, segundo Lopes (2001), passaram a buscar novas alternativas de exploração de açaí, com o objetivo de atender às expectativas atuais e futuras do mercado. Na realidade, os extrativistas foram impulsionados a praticarem o manejo dos açaizais nativos para produzirem mais, em resposta aos aumentos de preços (Santana \& Costa, 2008; Santana \& Santana, 2008).

O manejo do açaizal na várzea não foi suficiente para atender à demanda, o que deu início a uma nova modalidade de se produzir açaí, por meio do cultivo em terra firme (Nogueira, 2011). Esse sistema de produção tem evoluído no Estado do Pará, onde o cultivo é praticado seguindo-se recomendações técnicas de espaçamento, adubação, irrigação e plantas melhoradas geneticamente (Santana et al., 2010; Farias Neto et al., 2011; Nogueira et al. 2013), que portanto, são mais produtivas e precoces, o que tem proporcionado uma produtividade média das áreas plantadas da ordem de $15 \mathrm{t} / \mathrm{ha}$ a $20 \mathrm{t} / \mathrm{ha}$ (IBGE, 2006).
Contudo, essa expansão da produção de fruto não conseguiu equilibrar o mercado, uma vez que a procura externa aumentou e a oferta não acompanhou essa expansão (Santana, 2004; Santana \& Costa, 2008). Esse processo de expansão da produção do açaí-fruto está beneficiando os produtores extrativistas e os consumidores, assim como contribuindo para eliminar os impactos ambientais da extração predatória do palmito, que passa a ser extraído apenas das plantas improdutivas das áreas de plantios e de manejo, quando, antes todas as plantas eram cortadas, pois o palmito era mais valorizado do que o fruto. Porém, a taxa de distribuição desses resultados para a sociedade ainda não é conhecida.

Até o momento, foram identificados apenas os trabalhos de Lopes (2001), Lopes et al. (2002), Silva et al. (2006), visando a calcular os retornos sociais do cultivo do açaí. Estes estudos compreendem o período de 1980 a 2000 , envolvendo apenas a produção extrativista de frutos de açaí, uma vez que as técnicas de manejo e os plantios em terra firme, sem e com irrigação, ganharam expressão apenas nesta última década.

Este trabalho objetiva calcular os retornos socioeconômicos gerados com a adoção de tecnologias de manejo, nas áreas de várzea e de plantios de açaí em terra firme, e sua distribuição entre os consumidores e produtores paraenses, no período de 2001 a 2009.

\section{MATERIAL E MÉTODOS}

\section{Área de estudo e dados utilizados}

A área de estudo foi o Estado do Pará, por ser o maior produtor nacional de açaí e liderar as mudanças tecnológicas no processo produtivo (Nogueira, 2011).

Os dados de produção e preço, referentes ao período de 1994 a 2009, foram obtidos junto ao Instituto Brasileiro de Geografia e Estatística (IBGE, 2006). As informações de salário rural, renda per capita e o Índice Geral de Preços - Disponibilidade Interna (IGP-DI) foram obtidas da revista Conjuntura Econômica, da Fundação Getúlio Vargas (FGV). O conjunto destas informações propiciou a estimação das equações de demanda e oferta do açaífruto, conforme Nogueira (2011). A partir desses resultados, determinou-se o valor do benefício socioeconômico oriundo da utilização de tecnologias no sistema de produção de açaí, bem como sua distribuição entre consumidores e produtores.

A metodologia de cálculo dos impactos socioeconômicos da adoção de tecnologias é universalmente consolidada e envolve a aplicação do conceito de excedente econômico total ou benefício social líquido total, desenvolvido por Marshall (1982). Este conceito contempla o excedente do produtor, ou lucro do produtor, e o excedente do consumidor, ou benefício social do consumidor, 
gerado pelas novas tecnologias e inovações implantadas e é o único que incorpora a dimensão social no cálculo dos benefícios e custos sociais.

Assim, quando a análise envolve um único produto, ou atividade produtiva, como é o caso deste trabalho, o excedente econômico total é o método adequado para a determinação dos benefícios socioeconômicos e os retornos sociais de investimento em pesquisa, desenvolvimento e implantação de novas tecnologias. Este método pode ser aplicado para medir os retornos socioeconômicos e, ou, ambientais bruto e líquido ex-ante, ou retornos potenciais ex-post, ou retornos reais, como é o caso deste trabalho, dado que as tecnologias estão em processo de consolidação.

Nesta perspectiva, a pioneira aplicação foi realizada por Grilliches (1958), para medir os benefícios sociais ex-ante da descoberta do milho híbrido. O modelo adotou casos extremos para as elasticidades-preço da demanda e da oferta, por dificuldade de dados para estimar empiricamente estes parâmetros. A segunda aplicação do conceito de excedente econômico foi realizada por Peterson (1967), para estimar o retorno social da pesquisa com aves. O autor adotou oferta inclinada e deslocamento paralelo. Em seguida, Lindner e Jarrett (1978) propôs uma forma mais adequada para medir o excedente econômico, assumindo que o deslocamento da oferta deve ser proporcional e divergente, dado que o processo de adoção das novas tecnologias não ocorre de forma instantânea e os impactos na redução do custo ou no aumento de produtividade não são homogêneos. Depois, Santana \& Khan (1987) aplicaram esta metodologia para medir os retornos sociais da adoção de tecnologias pelos produtores de feijão caupi, na região nordeste. Também Santana \& Khan (1992) aplicaram, de forma pioneira, essa metodologia para calcular os custos da depredação da castanha-do-brasil, no Estado do Pará. Mais recentemente, Angelo et al. (2013) aplicaram a metodologia utilizada por Santana para estimar o impacto do desmatamento das reservas de castanheiras na Amazônia. Por fim, todos os cálculos realizados por esses autores foram realizados com base em modelos lineares de demanda e oferta. Assim, a aplicação desses modelos a casos em que as equações de demanda e de oferta e demanda são especificadas na forma não linear pode gerar viés de super ou de subestimação do benefício socioeconômico.

Ayer e Schuh (1972) e Avila (1981) assumiram um deslocamento pivotal da curva de oferta, especificada na forma logarítmica, para estimar o benefício socioeconômico da pesquisa com algodão, em São Paulo, e com arroz no Rio Grande do Sul. O coeficiente de deslocamento da curva de oferta foi definido pela variação entre a produção potencial e a produção obtida antes dos resultados da pesquisa. Assim, para evitar viés de mensuração, o benefício socioeconômico foi calculado por meio de integral.
Por fim, Nogueira (2011) especificou o modelo de mercado, na forma logarítmica, para estimar o benefício socioeconômico da mudança tecnológica de manejo e cultivo racional do açaí no Estado do Pará, por meio do cálculo integral. Para isto, considerou-se o deslocamento da oferta na forma proporcional divergente, dado que essas tecnologias proporcionaram aumentos da produção e da produtividade do açaí. Com isto, os resultados refletem os valores reais dos benefícios socioeconômicos obtidos pelos produtores e pelos consumidores de açaí do Estado do Pará.

Os parâmetros das equações de demanda e de oferta de açaí-fruto foram estimados por Nogueira et al. (2013), a partir dos dados do IBGE, do SIMA/SAGRI e da Revista Conjuntura Econômica, da Fundação Getúlio Vargas. O método de estimação do sistema de equações simultâneas foi o de Momentos Generalizados (MMG), por gerar estimativas consistentes e eficientes para os parâmetros do modelo.

\section{Determinação do Benefício Socioeconômico Total (BST)}

Este estudo utilizou o conceito de excedente econômico, estimado com base no conhecimento das equações de demanda e de oferta, a partir das quais se definem os excedentes do consumidor e do produtor, cujos valores e distribuições dependem da magnitude das elasticidadespreço da demanda e da oferta (Nogueira, 2011).

A utilização de novas tecnologias de manejo e de irrigação tende a gerar externalidades positivas, mediante aumento de produtividade e das oportunidades de emprego e renda para a economia local (Nogueira, 2011). Os benefícios econômicos e sociais gerados pela implantação dessas tecnologias nos sistemas de produção do açaí fruto são diversos, podendo ser adequadamente medidos pela variação dos excedentes dos consumidores e dos produtores.

A área abaixo da linha de demanda, que representa o benefício marginal social (BMS), e acima do preço de equilíbrio $\left(\mathrm{CB}_{1} \mathrm{P}_{\mathrm{e}}\right)$, é o excedente do consumidor (EC) (Figura 1) e representa a diferença entre o que o consumidor está disposto a pagar por determinado produto e o que efetivamente paga, gerando, como resultado da compra, um excedente de satisfação (Varian, 1994).

Com efeito, o excedente do produtor é a soma das diferenças entre o preço de mercado e o custo marginal de produção, relativo a todas as unidades produzidas (Pindyck \& Rubinfeld, 2002). Ou seja, o excedente do produtor é uma medida de compensação, excedente ao valor que é pago aos fornecedores dos fatores de produção empregados no processo produtivo e, quanto maior o excedente do produtor, maior será o seu bem-estar; na Figura 1 , é a área situada abaixo da linha de preço de equilíbrio 
do mercado e acima da curva de oferta, delimitada pelos pontos $\left(\mathrm{A}_{1} \mathrm{~B}_{1} \mathrm{P}_{\mathrm{e}}\right)$.

A situação de equilíbrio entre demanda e oferta define a quantidade e o preço que maximizam o bem-estar econômico dos produtores e consumidores e, segundo Santana (2005), é a soma dos excedentes do consumidor e produtor, que, para um mercado em concorrência pura, representa a máxima eficiência alocativa deste mercado. Assim, o excedente econômico é representado, na Figura 1, como a área compreendida entre as linhas de demanda e de oferta de mercado.

O excedente econômico do mercado de açaí é representado pela soma dos excedentes do consumidor e do produtor $(\mathrm{EC}+\mathrm{AP})$, constituído, inicialmente, sem a influência das tecnologias que foram implantadas no processo produtivo.

Assumindo-se que as equações de demanda e de oferta, que estão representadas na Figura 1, sejam especificadas didaticamente pelas equações abaixo:

Demanda: $P=a-b Q$

Oferta: $P=c+d Q$

em que P é o preço do açaí ( $\mathrm{R} \$ / \mathrm{t}), \mathrm{Q}$ a quantidade de açaí (t) e (a, b, c e d) os parâmetros a serem estimados pelo método de equações simultâneas, conforme Santana (2003) e Nogueira (2011), tem-se que os excedentes dos consumidores e dos produtores podem ser calculados por meio da aplicação do cálculo integral, da seguinte forma:

Excedente do consumidor:

$E C=\int_{0}^{Q e}(a-b Q) d q-P_{\theta} \cdot Q_{\theta}$

Excedente do produtor:

$E P=P_{\theta} \cdot Q_{\theta}-\int_{0}^{Q \theta}(c+d Q) d q$
Excedente econômico:

$E E=\int_{0}^{Q e}(a+b Q) d q+\int_{0}^{Q e}(c+d Q) d q$

Adotando-se a hipótese de que a utilização das tecnologias de manejo dos açaizais da várzea, a tecnologia de irrigação em diversos sistemas (monocultivo, consórcio e agroflorestal), que incorporam as inovações tecnológicas de processo, contribuiu, efetivamente, para o aumento da produção de açaí, a partir de 2002. Esse aumento de produção gera um deslocamento da curva de oferta, produzindo um novo equilíbrio de mercado, em que os excedentes dos consumidores e dos produtores devem aumentar, e, em consequência, o benefício socioeconômico total para a sociedade.

As tecnologias podem induzir mudanças na oferta de diversas formas, como proposto por Lindner \& Jarrett (1978). As formas mais comuns são os deslocamentos paralelos e pivotais. Para o açaí, adota-se a hipótese de que o deslocamento da oferta foi pivotal divergente, em função de que a produtividade do açaí muda com as tecnologias de manejo e de irrigação, sendo mais forte a mudança no custo marginal social para os extrativistas que adotaram a tecnologia, do que para os pequenos produtores que já cultivavam o açaí manejado. Na Figura 1, é representado o mercado atual de açaí, com a incorporação do conjunto de tecnologias utilizadas no processo produtivo. A linha de oferta $\left(\mathrm{S}_{1}\right)$ representa o custo marginal social dos produtores que estão fazendo manejo e utilizando novas tecnologias (CMSt).

O benefício socioeconômico total (BST), gerado pelas mudanças tecnológicas no sistema de produção do açaí, está representado pela área entre as duas linhas de oferta e abaixo da linha de demanda $\left(\mathrm{A}_{1} \mathrm{~B}_{1} \mathrm{~B}_{2} \mathrm{~A}_{2}\right)$. De acordo com Alston et al. (1995), esta área pode ser vista como a soma de duas partes: a redução dos custos sobre a quantidade

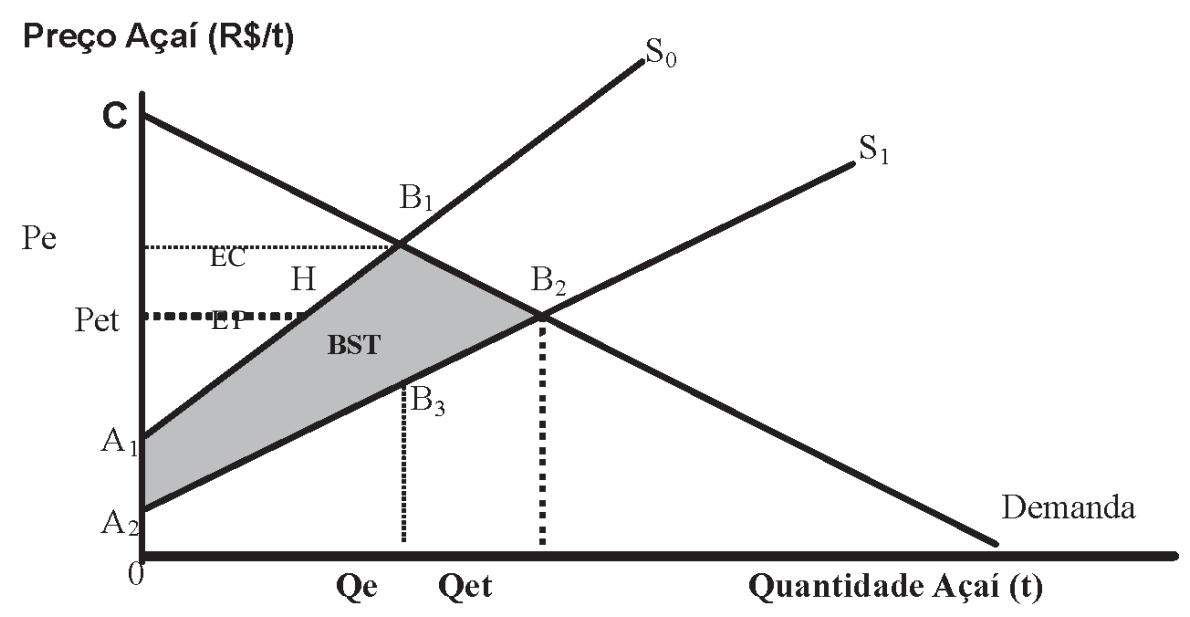

Figura 1: Representação esquemática do excedente do consumidor e do produtor de açaí, considerando-se todas as inovações introduzidas no processo produtivo do açaí.

Rev. Ceres, Viçosa, v. 63, n.1, p. 001-007, jan-fev, 2016 
original (a área entre as duas curvas de oferta a esquerda de $\mathrm{Q}_{\mathrm{e}}$ ) e o excedente econômico devido ao incremento da produção e do consumo (área triangular $\mathrm{B}_{1} \mathrm{~B}_{2} \mathrm{~B}_{3}$, o valor do incremento ao consumo, menos o custo total do incremento da produção).

Esta área pode ser obtida aplicando-se a técnica do cálculo integral, conforme Nogueira (2011). Antes disso, a nova linha de oferta deve ser especificada. Um fator de deslocamento da oferta é definido como k, que representa a relação entre a produção obtida com as novas tecnologias e a obtida com o extrativismo. Assim, o novo equilíbrio de mercado deve ser representado da seguinte forma:

Demanda: $P=a-b Q$

Oferta: $P=k+(c+d Q)$

em que P é o preço do açaí (R\$/t), Q a quantidade de açaí (t), (a, b, c e d) os parâmetros a serem estimados pelo método de equações simultâneas, conforme Santana (2003), e k é o deslocador da oferta, dado por:

$k=\left(1-\frac{Q_{e}}{Q_{e t}}\right)$, em que $\mathrm{Q}_{\mathrm{e}}$ é a quantidade de açaí obtida no sistema extrativo e $\mathrm{Q}_{\mathrm{et}}$ a quantidade de açaí por hectare obtida nos sistemas com inovação tecnológica.

Assim, o benefício socioeconômico total (BST) produzido é dado pela equação abaixo:

$$
\begin{aligned}
& B S T=\int_{0}^{Q_{\theta}}(c+d Q) d q+\int_{Q_{e}}^{Q_{\theta t}}(a-b Q) d q- \\
& \int_{0}^{Q_{\theta} t}(k+c+d Q) d q
\end{aligned}
$$

As estimativas dos parâmetros das equações de demanda e de oferta foram obtidas a partir de Nogueira (2011), que ajustou um modelo simultâneo de oferta e demanda pelo método dos momentos generalizados, de onde se obtiveram as elasticidades-preço da demanda e da oferta de açaí fruto no Estado do Pará.

\section{RESULTADOS E DISCUSSÃO}

As equações de demanda e de oferta foram estimadas por Nogueira et al. (2013). Neste estudo, as funções de demanda e oferta foram definidas, respectivamente, da seguinte forma:

Equação de Demanda: $\ln Q A C^{D}=17,684-0,779 \ln P a$

Equação de Oferta: $\ln Q A C^{S}=6,902+0,7937 \ln P a$

O coeficiente de elasticidade-preço da demanda foi de $-0,779$, indicando que se trata de um produto inelástico a preço, ou seja, elevações de preços tendem a provocar uma redução menos que proporcional nas quantidades demandadas. Assim, quando os preços variam 10\%, as quantidades tendem a variar 7,79\% em sentido contrário, ceteris paribus (Nogueira et al., 2013). Este resultado está em conformidade com outros trabalhos já desenvolvidos por Lopes \& Santana (2005), que estimaram a elasticidade-preço para o açaí, para o produtor, em -0,515, e por Santana \& Gomes (2005) que estimaram para o mercado atacadista de açaí, em -0,825.

Segundo Nogueira (2011), a elasticidade-preço da oferta de açaí-fruto foi de 0,937 , indicando que, para cada incremento de $10 \%$ no preço real do açaí, a quantidade ofertada tende a aumentar $9,37 \%$, ceteris paribus. Isto significa que o açaí é um produto de oferta inelástica a preço, ou seja, a quantidade ofertada varia menos que proporcionalmente às mudanças no preço, o que também tende a produzir forte instabilidade da renda dos produtores, diante de aumentos nas quantidades ofertadas no período da safra. Com o passar do tempo, a oferta tornouse mais elástica, pois Lopes \& Santana (2005) encontraram um coeficiente de elasticidade de 0,517 , e Santana \& Gomes (2005) de 0,596, bem inferiores ao encontrado neste estudo.

A elasticidade de ajustamento, associada à variável QAÇ $_{\mathrm{t}-1}$, mostrou-se significativa a 1\%, evidenciando bons resultados de produção atual. A elasticidade de ajustamento da oferta de curto prazo do açaí foi de 0,706 , bem inferior ao coeficiente de elasticidade-preço em longo prazo, que foi de 1,327, o qual é elemento deslocador da oferta $(\mathrm{K})$.

\section{Avaliação do benefício socioeconômico da utilização de novas tecnologias na produção de açaí}

O Beneficio Socioeconômico Total (BST) proveniente da adoção de tecnologias no sistema de produção de açaí, tomadas a partir de 2001, foi calculado a partir das estimativas das equações de demanda e de oferta. Para isso, considerou-se o preço de equilíbrio $\left(\mathrm{P}_{\mathrm{e}}\right)$, igual a $\mathrm{R} \$ 535,39$, e quantidade de equilíbrio $\left(\mathrm{Q}_{\mathrm{e}}\right)$, igual a 358.254,89 t.

A partir das elasticidades-preço da oferta foi possível estimar o deslocador da oferta $\mathrm{K}$, a longo prazo, cujo valor foi de 1,327. Esse deslocador foi encontrado por meio da divisão da elasticidade-preço da oferta $(0,937)$ pela elasticidade de ajustamento da oferta de curto prazo $(0,706)$. Assim, foi possível calcular os excedentes dos consumidores e produtores, antes e depois adoção de tecnologia, por meio da aplicação do cálculo integral, apresentada na Tabela 1.

Com base nos resultados (Tabela 1) constatou-se que o benefício socioeconômico total gerado por ano, ao longo do período de 1994 a 2009, para a população paraense, antes da adoção da tecnologia, foi de R $\$ 13.195,50$ mil. Os 
Tabela 1: Resultados da análise dos benefícios socioeconômicos gerados anualmente, antes e depois da adoção de novas tecnologias no sistema de produção de açaí fruto, no Estado do Pará - 1994-2009

\begin{tabular}{lccc}
\hline & Antes da tecnologia & Com tecnologia & Expansão do consumo \\
\hline Excedente Consumidor $(\mathrm{R} \$ 1.000,00)$ & $6.848,55$ & $18.515,46$ & $33.367,60$ \\
Excedente do Produtor $(\mathrm{R} \$ 1.000,00)$ & $6.346,95$ & $7.248,33$ & $31.276,88$ \\
Excedente Econômico $(\mathrm{R} \$ 1.000,00)$ & $13.195,50$ & $25.763,79$ & $64.644,48$ \\
\hline
\end{tabular}

Fonte: Resultados da pesquisa.

benefícios para os consumidores foram de $\mathrm{R} \$ 6.848,55$ mil, cerca de $51,9 \%$ dos benefícios totais. Quanto aos produtores, esses obtiveram o retorno de $\mathrm{R} \$ 6.346,95$ mil, o equivalente a $48,09 \%$. Comparativamente, os benefícios gerados aos produtores (medidos pelo excedente do produtor) foram inferiores aos benefícios dos consumidores (medidos pelo excedente do consumidor).

As novas tecnologias produziram incremento de produção de açaí no Estado, evidenciado no deslocamento da curva de oferta para a direita e para baixo, aumentando simultaneamente os excedentes do consumidor e do produtor. A magnitude dos benefícios anuais gerados para a sociedade paraense foi da ordem $\mathrm{R} \$ 25.763,79$ mil. Os consumidores foram os principais beneficiados, com R\$ $18.515,46$ mil, cerca de 71,86\% dos retornos totais. Quanto aos produtores, esses se apropriaram da menor parcela, um montante de $\mathrm{R} \$ 7.248,33$ mil, ou seja, $28,13 \%$ do benefício total. Esse resultado decorre da mudança da inclinação das curvas de oferta e demanda, uma vez que, pelos resultados obtidos, a demanda apresentou comportamento inelástico a preço, e a oferta tornou-se menos inelástica a preço $(0,937)$ e, nestas condições de oferta mais elástica do que a demanda, tem-se um cenário mais favorável aos consumidores do que aos produtores. Desse modo, observou-se que a adoção da tecnologia (manejo de açaizais nativos e cultivo de açaí em terra firme) aumentou o bemestar da população paraense, formada por consumidores e produtores.

Analisou-se ainda a expansão do consumo de açaí no Estado do Pará, no período de 2001 a 2009. Para isso, utilizou-se o coeficiente da variável dummy da equação de demanda, cujo valor foi de 0,669 , o qual foi somado com um $(0,669+1=1,669)$ e, posteriormente, multiplicado pela equação de demanda, dando origem a uma nova equação de demanda para efeito de captar as variações do consumo do fruto no Estado.

Com base nos resultados da (Tabela 1), constatou-se que, a partir de 2001, houve uma elevação no consumo de açaí, representada pelo deslocamento da curva de demanda para cima e para a direita, gerando um novo equilíbrio entre preço $(R \$ 1.704,45)$ e quantidade $(352.654 .717,9 t)$.

Os benefícios socioeconômicos gerados com a expansão do consumo somaram o montante de $\mathrm{R} \$ 64.644,48$ mil, favorecendo mais os consumidores do que os produto- res. Os consumidores apropriaram-se de $\mathrm{R} \$ 33.367,60 \mathrm{mil}$, ou seja, $51,61 \%$ do benefício total, restando aos produtores, o equivalente a $48,38 \%$ dos benefícios, representando $\mathrm{R} \$ 31.276,88$ mil.

O aumento da expansão do consumo de açaí fez incrementar significativamente a produção do fruto, no Estado do Pará. Nesse contexto, vale destacar que o percentual de consumo de açaí no mercado paraense devese, simultaneamente, à melhoria da renda da população, à agregação de valor ao produto e, ainda, à diversificação das linhas de produção das indústrias de polpas de frutas, que visam a atender de forma adequada às necessidades dos consumidores nacionais e estrangeiros.

\section{CONCLUSÕES}

No período analisado, com a adoção de tecnologia no sistema de produção de açaí, houve substancial aumento do benefício socioeconômico para a população paraense, atingindo o patamar de $\mathrm{R} \$ 25.763,79$ mil, apresentando um incremento de $48,78 \%$, em relação ao benefício obtido antes da adoção da tecnologia. Os consumidores foram os maiores beneficiados, com 71,86\% dos benefícios totais.

Ademais, a expansão do consumo de açaí contribuiu para aumento do benefício socioeconômico gerado para a população paraense, com o patamar de $\mathrm{R} \$ 64.644,48$ mil, sendo que ambos os agentes de mercado beneficiaram-se de forma equitativa dos benefícios gerados.

Assim, tanto a adoção de novas tecnologias no sistema de produção de açaí-fruto quanto a expansão do consumo do fruto nos mercados nacional e internacional têm contribuído para a melhoria do bem-estar da população paraense, que vende e consome o fruto no Estado do Pará.

\section{REFERÊNCIAS}

Alston JM, Norton GW \& Pardey PG (1995) Science under scarcity: principles and practice for agricultural research evaluation and priority setting. Ithaca, ISNAR. 585p.

Angelo H, Pompermayer RS, Almeida AN \& Moreira JMMAP (2013) O custo social do desmatamento da Amazônia brasileira: o caso da castanha-do-brasil (Berthellotia excelsa). Ciência Florestal, 23:183-191.

Avila AFD (1981) Evaluation de la recherche agronomique au Brésil: le cas de la recherche rizicole de I'IRGA au Rio Grande do Sul. Tese de doutorado. Université de Montpellier, France. 217p. 
Ayer HW \& Schuh E (1972) Social rates of return and other aspects of agricultural research in São Paulo, Brazil. American Journal of Agricultural Economics, 54:557-569.

Bobbio FO, Druzian JI, Abrão PA, Bobbio PA \& Fadelli S (2000) Identificação e quantificação das antocianinas do fruto do açaizeiro (Euterpe oleracea Mart.). Ciência e Tecnologia de Alimentos, 20:388-390.

Farias Neto JT de, Resende MDV de \& Oliveira MSP de (2011) Seleção simultânea em progênies de açaizeiro irrigado para produção e peso do fruto. Revista Brasileira de Fruticultura, 33:532539 .

Grilliches Z (1958) Research costs and social returns: hybrid corn and related innovations. Journal of Political Economy, 66:419431

Homma AKO \& Santana AC de (2009) A agroindústria na Região Norte. In: Zibetti DW \& Barroso LA (Eds.) Agroindústria: uma análise no contexto socioeconômico e jurídico brasileiro. São Paulo, LEUD. p.19-43.

IBGE (2006) Censo Agropecuário de 2006. Rio de Janeiro, IBGE p.1-777.

IBGE (2013) Produção Agrícola Municipal, 1980 - 2013. Disponível em: <http://www.sidra.ibge.gov.br> Acessado em: 10 de setembro de 2013.

Lindner RK \& Jarret FG (1978) Supply Shift and The Size Off Research Benefit. American Journal of Agricultural Economics, $60: 48-58$

Lopes LMB (2001) Mercado e distribuição dos retornos sociais do manejo do açaí para produção de fruto. Dissertação de Mestrado. Belém, Universidade da Amazônia. 73p.

Lopes MLB \& Santana AC de (2005) O mercado do fruto do Açaizeiro (Euterpe Oleracea Mart.) no estado do Pará. In: Carvalho DF (Org.) Economia da Amazônia nos anos 90. Belém, Universidade da Amazônia. p.65-84.

Lopes MLB, Santana AC de, Homma AKO \& Nogueira OL (2002) Benefícios sociais do açaí manejado no Estado do Pará. In: Congresso Brasileiro de Economia e Sociologia rural, Passo Fundo-RS. Anais, SOBER. p.1-12.

Marshall A (1982) Princípios de economia. São Paulo, Abril Cultural. $272 \mathrm{p}$.

Nogueira AKM (2011) As tecnologias utilizadas na produção de açaí e seus benefícios socioeconômicos no estado do Pará. Dissertação de Mestrado. Universidade Federal Rural da Amazônia, Belém. 73p.

Nogueira AKM, Santana AC de \& Garcia WS (2013) A dinâmica do mercado de açaí fruto no Estado do Pará: de 1994 a 2009. Revista Ceres, 60:324-331.

Peterson WL (1967) Returns to poultry research in the United States. Journal of Farm Economics, 49:656-670.

Pindyck RS \& Rubinfeld DL (2002) Microeconomia. 5 ed. São Paulo, Prentice Hall. 711p.
Santana AC de (2003) Métodos quantitativos em economia: elementos e aplicações. Belém, UFRA. 484p.

Santana AC de \& Costa FA (2008) Mudanças recentes na oferta e demanda do açaí no Estado do Pará. In: Santana AC de, Carvalho DF \& Mendes AFT (Eds.) Análise sistêmica da fruticultura paraense: organização, mercado e competitividade empresarial. Belém, Banco da Amazônia. p.205-226.

Santana AC de \& Gomes SC (2005) Mercado, comercialização e ciclo de vida do mix de produtos do açaí no Estado do Pará. In: Carvalho DF (Org.) Ensaios selecionados sobre a economia da Amazônia nos anos 90. Belém, Universidade da Amazônia. p.225-278.

Santana AC de \& Khan AS (1987) Avaliação e distribuição dos ganhos sociais da adoção de novas tecnologias na cultura de feijão caupi no Nordeste. Revista de Economia e Sociologia Rural, 25:191-203.

Santana AC de \& Khan AS (1992) Custo social da depredação florestal no Pará: o caso da castanha-do-brasil. Revista de Economia e Sociologia Rural, 30:253-269.

Santana AC de \& Santana RL (2008) Dinâmica e sustentabilidade do arranjo produtivo local da fruticultura na Amazônia. Frutal Amazônia, 3:01-14.

Santana AC de (2004) Análise do desempenho competitivo das agroindústrias de polpa de frutas do estado do Pará. Revista de Economia e Agronegócio, 2:495-523.

Santana AC de (2005) Elementos de economia, agronegócio e desenvolvimento local. Belém, UFRA. 197p.

Santana AC de (2007) Índice de desempenho competitivo das empresas de polpa de frutas do Estado do Pará. Revista de Economia e Sociologia Rural, 45:749-775.

Santana AC de, Carvalho DF \& Mendes FAT (2010) Organização e competitividade das empresas de polpa de frutas do Estado do Pará: 1995 a 2004. Belém, Unama. 176p.

Silva IM da \& Silva FM da (2006) Perfil do consumidor domiciliar de açaí na Região Metropolitana de Belém - PA. In: XLIV Congresso da Sociedade Brasileira de Economia e Sociologia Rural, Fortaleza. Anais, Sociedade Brasileira de Economia e Sociologia Rural. p.01-15.

Silva IM da, Santana AC de \& Reis MS (2006) Análise dos retornos sociais oriundos de adoção tecnológica na cultura do açaí no Estado do Pará. Revista Amazônia: Ciência \& Desenvolvimento, 2:25-37.

Teixeira LN, Stringheta PC \& Oliveira FA de (2008) Comparação de métodos para quantificação de antocianinas. Revista Ceres, 55:297-304.

Varian H (1994) Microeconomia: Princípios Básicos. 2a ed. Rio de Janeiro, Campus. 710p. 